Pacific Journal of Mathematics

ON CHARACTERIZING THE GAMMA AND THE NORMAL 


\section{ON CHARACTERIZING THE GAMMA AND THE NORMAL DISTRIBUTION}

\section{IGNACY KOTLARSKI}

We will characterize the gamma distribution by the nature of the joint distribution of the two quotients $X_{1} / X_{3}, X_{2} / X_{3}$ for three identically gamma distributed random variables.

It is well known that if two independent identically distributed random variables $X_{1}, X_{2}$ have the gamma distribution given by the density

$$
f(x)=\left\{\begin{array}{lll}
0 & \text { for } x \leqq 0 \\
\frac{a^{p}}{\Gamma(p)} x^{p-1} e^{-a x} & \text { for } x>0 & \left(\begin{array}{l}
a>0 \\
p>0
\end{array}\right)
\end{array}\right.
$$

then their quotient

$$
Y=X_{1} / X_{2}
$$

has the beta distribution of the second kind given by the density

$$
g(y)= \begin{cases}0 & \text { for } y \leqq 0 \\ \frac{1}{B(p, p)} \cdot \frac{y^{p-1}}{(1+y)^{2 p}} & \text { for } y>0 .\end{cases}
$$

However, this property does not characterize the gamma distribution uniquely. There exist pairs of independent positive identically distributed random variables $X_{1}, X_{2}$ whose common distribution function $F(x)$ differs from the one given by the density (1), but where the quotients (2) are distributed according to the density (3). Some such distribution functions $F(x)$ are given by the following densities

$$
\begin{aligned}
& f_{1}(x)= \begin{cases}0 & \text { for } x \leqq 0 \\
\frac{a^{p}}{\Gamma(p)} x^{-p-1} e^{-a / x} & \text { for } x>0\end{cases} \\
& f_{2}(x)= \begin{cases}0 & \text { for } x \leqq 0 \\
\frac{2 \Gamma\left(\frac{2 p+1}{2}\right)}{\Gamma\left(\frac{p}{2}\right) \Gamma\left(\frac{p+1}{2}\right)} \cdot \frac{x^{p-1}}{\left(1+x^{2}\right)^{p+1 / 2}} & \text { for } x>0\end{cases}
\end{aligned}
$$




$$
f_{3}(x)= \begin{cases}0 & \text { for } x \leqq 0 \\ \frac{2 \Gamma\left(\frac{2 p+1}{2}\right)}{\Gamma\left(\frac{p}{2}\right) \Gamma\left(\frac{p+1}{2}\right)} \cdot \frac{x^{p}}{\left(1+x^{2}\right)^{p+1 / 2}} & \text { for } x>0 .\end{cases}
$$

Now let $X_{1}, X_{2}, X_{3}$, be three independent positive identically distributed random variables whose common distribution function is $F(x)$. It is interesting that although the distribution of one quotient (2) does not characterize $F(x)$ uniquely, the joint distribution of two such quotients

$$
Y_{1}=X_{1} / X_{3}, Y_{2}=X_{2} / X_{3}
$$

characterize (by some assumptions) $F(x)$ uniquely, up to a change of the scale.

The condition of identical distributions of $X_{1}, X_{2}, X_{3}$ may be omitted. The distribution functions $F_{k}(x)$ of the independent positive random variables $X_{k}(k=1,2,3)$ are uniquely characterized (by some assumptions) by the joint distribution of the pair of qoutients (5), up to a change of the scale.

The normal distribution is also characterized in the same way. There are also some generalizations of these problems.

\section{Some lemmas.}

Lemma 1. Let $X_{1}, X_{2}, X_{3}$ be three independent real random variables, and let

$$
Z_{1}=X_{1}-X_{3}, Z_{2}=X_{2}-X_{3} .
$$

If the characteristic function of the pair $\left(Z_{1}, Z_{2}\right)$ does not vanish, then the distribution of $\left(Z_{1}, Z_{2}\right)$ determines the distributions of $X_{1}$, $X_{2}, X_{3}$ up to a change of the location.

Proof. Denote $\varphi\left(t_{1}, t_{2}\right)$ the characteristic function of the pair $\left(Z_{1}, Z_{2}\right)$, and $\varphi_{k}(t)$ the characteristic functions of $X_{k}(\mathrm{k}=1,2,3)$. Then there is

$$
\begin{aligned}
\varphi\left(t_{1}, t_{2}\right) & =E \exp \left[i\left(t_{1} Z_{1}+t_{2} Z_{2}\right)\right. \\
& =E \exp \left[i\left[t_{1}\left(X_{1}-X_{3}\right)+t_{2}\left(X_{2}-X_{3}\right)\right]\right] \\
& =E \exp \left[i\left[t_{1} X_{1}+t_{2} X_{2}+\left(-t_{1}-t_{2}\right) X_{3}\right]\right] \\
& =\varphi_{1}\left(t_{1}\right) \varphi_{2}\left(t_{2}\right) \varphi_{3}\left(-t_{1}-t_{2}\right) .
\end{aligned}
$$

The condition of nonvanishing of $\varphi\left(t_{1}, t_{2}\right)$ is equivalent to nonvanishing of any of the functions $\varphi_{k}(t)(\mathrm{k}=1,2,3)$. 
Let $U_{1}, U_{2}, U_{3}$ be another three independent real random variables, having characteristic functions $\psi_{k}(t)=E e^{i t U_{k}}(k=1,2,3)$ and satisfying the assumptions of Lemma 1 . Let $V_{1}=U_{1}-U_{3}, V_{2}=U_{2}-U_{3}$ and $\psi\left(t_{1}, t_{2}\right)=E \exp \left[i\left(t_{1} V_{1}+t_{2} V_{2}\right)\right]$. Making the same considerations as for formula (7) it is easy to see that there is

$$
\psi\left(t_{1}, t_{2}\right)=\psi_{1}\left(t_{1}\right) \psi_{2}\left(t_{2}\right) \psi_{3}\left(-t_{1}-t_{2}\right) .
$$

Let the pairs $\left(Z_{1}, Z_{2}\right)$ and $\left(V_{1}, V_{2}\right)$ have the same distribution. Then their characteristic functions are equal. Using formulae (7) and (8) one can obtain the following equation

$$
\begin{aligned}
\psi_{1}\left(t_{1}\right) \psi_{2}\left(t_{2}\right) \psi_{3}\left(-t_{1}-t_{2}\right)=\varphi_{1}\left(t_{1}\right) \varphi_{2}\left(t_{2}\right) \varphi_{3}\left(-t_{1}-t_{2}\right) \\
\left(-\infty<t_{1}<+\infty,-\infty<t_{2}<+\infty\right) .
\end{aligned}
$$

Put

$$
\psi_{k}(t)=\varphi_{k}(t) \cdot p_{k}(t) \quad(k=1,2,3) .
$$

Putting (10) into the equation (9) one obtains the following equation

$$
p_{1}\left(t_{1}\right) p_{2}\left(t_{2}\right) p_{3}\left(-t_{1}-t_{2}\right)=1\left(\begin{array}{l}
-\infty<t_{1}<+\infty \\
-\infty<t_{2}<+\infty
\end{array}\right)
$$

in which $p_{k}(t)$ are unknown functions; they are complex functions, continuous on the whole line $-\infty<t<+\infty$, satisfying the condition

$$
p_{k}(0)=1 \quad(k=1,2,3) .
$$

In order to solve the equation (11) let us put $t_{1}=t, t_{2}=0$ and, later $t_{2}=t, t_{1}=0$. Then, using (12),

$$
p_{1}(t) \cdot p_{3}(-t)=1, \quad p_{2}(t) \cdot p_{3}(-t)=1 .
$$

Putting $p_{1}(t)$ and $p_{2}(t)$ given by (13) into the equation (11) and changing signs, we obtain

$$
p_{3}\left(t_{1}+t_{2}\right)=p_{3}\left(t_{1}\right) \cdot p_{3}\left(t_{2}\right) \text {. }
$$

The only continuous function which satisfies the equation (14) and condition (12) is the exponential function

$$
p_{3}(t)=e^{c t} \quad(-\infty<t<+\infty)
$$

where $c$ is a complex number. Putting (15) into (13) it is easy to see that

$$
p_{1}(t)=p_{2}(t)=p_{3}(t)=e^{c t} .
$$


Putting (16) into (10) there is

$$
\psi_{h}(t)=e^{c t} \rho_{k}(t) \quad(k=1,2,3) .
$$

Since the known property of characteristic functions $\varphi(-t)=\overline{\varphi(t)}$ the formulae (17) become

$$
\psi_{k}(t)=e^{i b t} \varphi_{k}(t) \quad(k=1,2,3)
$$

where $b$ is a real constant. This means that the distributions of $X_{k}$ are the same as of $U_{k}$ up to a change of the location.

Remark 1. The assumption of nonvanishing of the joint characteristic function of the differences (6) may be replaced by the assumption that all $X_{k}$ have analytic characteristic functions. All considerations are valid in such a case for $t$ complex, being inside a circle $|t|<t_{0}\left(t_{0}>0\right)$, where the characteristic functions do not vanish. Because of the analyticity of the characteristic functions the formulae (18) may be spread on the whole real line.

Remark 2. If the assumption of nonvanishing of the characteristic functions is omitted then the theorem becomes false. In order to show this the following example is given.

Example. Let $\left(X_{1}, X_{2}, X_{3}\right)$ and $\left(U_{1}, U_{2}, U_{3}\right)$ be two three's of independent real random variables having their characteristic functions $\varphi_{k}(t)$ and $\psi_{k}(t)$ respectively. Let be

$$
\left\{\begin{array}{l}
\varphi_{1}(t)=\varphi_{2}(t)=\psi_{1}(t)=\psi_{2}(t)=\left\{\begin{array}{cl}
0 & \text { for }|t|>1 \\
1-|t| & \text { for }|t| \leqq 1
\end{array}\right. \\
\varphi_{3}(t)=\left\{\begin{array}{cc}
0 & \text { for }|t|>2 \\
1-|t| / 2 \text { for }|t| \leqq 2
\end{array}\right. \\
\psi_{3}(t)=\varphi_{3}(t) \text { for }|t| \leqq 2, \psi_{3}(t+4)=\psi_{3}(t) .
\end{array}\right.
$$

It is easy to see that in such a case the equation (9) holds though $\psi_{3}(t)$ is not the same as $\varphi_{3}(t)$. Hence there exist trios $\left(X_{1}, X_{2}, X_{3}\right)$ and $\left(U_{1}, U_{2}, U_{3}\right)$ of independent real random variables whose distributions are not the same, but where the distributions of the corresponding pairs of differences $\left(X_{1}-X_{3}, X_{2}-X_{3}\right),\left(U_{1}-U_{3}, U_{2}-U_{3}\right)$ are the same.

Remark 3. Lemma 1 remains true if the three independent real random variables $\left(X_{1}, X_{2}, X_{3}\right)$ are replaced by $n$ such random variables $\left(X_{1}, X_{2}, \cdots, X_{n}\right)(n \geqq 3)$, and the pair of differences $\left(X_{1}-X_{3}, X_{2}-X_{3}\right)$ by $n-1$ differences $\left(X_{1}-X_{n}, X_{2}-X_{n}, \cdots, X_{n-1}-X_{n}\right)$. 
Remark 4. The differences in formula (6) may be replaced by sums.

Remark 5. Lemma 1 remains true if the real random variables $X_{k}$ are replaced by $n$-dimensional real random vectors.

Lemma 2. Let $X_{1}, X_{2}, X_{3}$ be three independent positive random variables, and let $\left(Y_{1}, Y_{2}\right)$ be their quotients given by formula (5). If the joint characteristic function of the pair (ln $Y_{1}, \ln Y_{2}$ ) does not vanish, then the distribution of $\left(Y_{1}, Y_{2}\right)$ determines the distributions of $X_{1}, X_{2}, X_{3}$ up to change of the scale.

Proof. The proof of Lemma 2 is obvious because $\ln X_{k}(k=1$, $2,3)$ satisfy the assumptions of Lemma 1.

REMARK 6. The positive random variables $X_{k}$ in Lemma 2 may be replaced by symmetrical about the origin real random variables satisfying the condition $P\left(X_{k}=0\right)=0$.

2. Characterizing the gamma distribution. The problem of characterizing the gamma distribution of two independent random variables $X_{1}, X_{2}$ by the distribution of their quotient was first posed by J. G. Mauldon [9], he showed that there is no such characterization. Further investigations on this problem were made by I. Kotlarski [1]. A full treatment of the problem has been made by R. G. Laha [6] (on this subject see also E. Lukacs, R. G. Laha [7], p. 59). The authors of [1] and [6] searched for the properties of the set of distribution functions $F(x)$ for which the quotient (2) is distributed according to (3), where $X_{1}, X_{2}$ are independent positive random variables identically distributed according to $F(x)$.

THEOREM 1. Let $X_{1}, X_{2}, X_{3}$ be three independent positive random variables, let $\left(Y_{1}, Y_{2}\right)$ be the pair of the quotients given by (5). The necessary and sufficient condition for $X_{k}$ to be gamma distributed with parameters $p_{k}$ and $a(a-$ common, $k=1,2,3)$ is that the joint distribution of $\left(Y_{1}, Y_{2}\right)$ is the bivariate beta distribution of the second kind given by the density

$$
g\left(y_{1}, y_{2}\right)=\left\{\begin{array}{ccc}
\frac{\Gamma\left(p_{1}+p_{2}+p_{3}\right)}{\Gamma\left(p_{1}\right) \Gamma\left(p_{2}\right) \Gamma\left(p_{3}\right)} \cdot \frac{y_{1}^{p_{1}-1} y_{2}^{p_{2}-1}}{\left(1+y_{1}+y_{2}\right)^{p_{1}+p_{2}+p_{3}}} \text { for } \begin{array}{l}
y_{1}>0 \\
y_{2}>0
\end{array} \\
\text { elsewhere } & &
\end{array}\right.
$$

Proof. The characteristic function of $\ln X_{k}$ where $X_{k}$ is gamma 
distributed with parameters $p_{k}$, $a$, is given by

$$
\varphi_{k}(t)=E e^{i t \ln x_{k}}=\alpha^{-i t} \frac{\Gamma\left(p_{k}+i t\right)}{\Gamma\left(p_{k}\right)} .
$$

The characteristic function of the pair $\left(\ln Y_{1}, \ln Y_{2}\right)$ is

$$
\begin{aligned}
\varphi\left(t_{1}, t_{2}\right) & =\varphi_{1}\left(t_{1}\right) \varphi_{2}\left(t_{2}\right) \varphi_{3}\left(-t_{1}-t_{2}\right) \\
& =\frac{\Gamma\left(p_{1}+i t_{1}\right)}{\Gamma\left(p_{1}\right)} \cdot \frac{\Gamma\left(p_{2}+i t_{2}\right)}{\Gamma\left(p_{2}\right)} \cdot \frac{\Gamma\left(p_{3}-i t_{1}-i t_{2}\right)}{\Gamma\left(p_{3}\right)}
\end{aligned}
$$

it is easy to see that the characteristic function of $\left(\ln Y_{1}, \ln Y_{2}\right)$ where $\left(Y_{1}, Y_{2}\right)$ is distributed according to the density (20) is also given by the right side of the formula (22), This ends the proof.

THEOREM 2. Let $X_{1}, X_{2}, X_{3}$ be three independent positive random variables, let $\left(U_{1}, U_{2}\right)$ be given by formulae

$$
U_{1}=\frac{X_{1}}{X_{1}+X_{2}}, \quad U_{2}=\frac{X_{1}+X_{2}}{X_{1}+X_{2}+X_{3}}
$$

The necessary and sufficient condition for $X_{k}$ to be gamma distributed with parameters $p_{k}$ and $a(a-$ common, $k=1,2,3)$ is that $U_{1}, U_{2}$ are independent beta distributed random variables, $U_{1}$ with parameters $\left(p_{1}, p_{2}\right)$, and $U_{2}$ with parameters $\left(p_{1}+p_{2}, p_{3}\right)$.

Proof. The necessary condition is obvious. In order to prove the sufficient condition let us put

$$
U_{1}=\frac{Y_{1}}{Y_{1}+Y_{2}}, \quad U_{2}=\frac{Y_{1}+Y_{2}}{1+Y_{1}+Y_{2}}
$$

where $Y_{1}$ and $Y_{2}$ are given by (5). It is easy to see that if $U_{1}, U_{2}$ are independent, $U_{1}$ being distributed accoring to the beta law of the first kind with the density

$$
g_{1}(u)= \begin{cases}\frac{1}{B\left(p_{1}, p_{2}\right)} u^{p_{1}-1}(1-u)^{p_{2}-1} & \text { for } 0<u<1 \\ 0 & \text { elsewhere }\end{cases}
$$

and $U_{2}$ has a similar distribution with parameters $\left(p_{1}+p_{2}, p_{3}\right)$, then $\left(Y_{1}\right.$, $Y_{2}$ ) is distributed according to the density (20). Using Theorem 1 it is easy to see that $X_{k}$ are gamma distributed with parameters $\left(p_{k}, a\right)$, $(a-$ common, $k=1,2,3)$. This ends the proof.

3. Characterizing the normal distribution. The problem of characterizing the normal distribution of two independent symmetrical about the origin random variables by the distribution of their quotient 
has been considered by several authors. J. G. Mauldon [9] showed that there is no such characterization. Further investigation on this problem were made by R. G. Laha $[3,4,5]$, I. Kotlarski [2], G. P. Steck [10].

Though there is no characterization of two independent symmetrical about the origin random variables by the distribution of their quotient, there is a characterization of three such random variables by the joint distribution of two their quotients. In this Section two theorems on characterizing the normal distribution in such a way are presented.

THEOREM 3. Let $X_{1}, X_{2}, X_{3}$ be three independent real symmetrical about the origin random variables satisfying the condition $P\left(X_{k}=0\right)=0, \quad(k=1,2,3)$. Let $\left(Y_{1}, Y_{2}\right)$ be the pair of quotients given by formula (5). The necessary and sufficient condition for $X_{k}$ to be normal distributed with a common standard deviation $\sigma$ is that the joint distribution of $\left(Y_{1}, Y_{2}\right)$ is the bivariate Cauchy distribution given by the density

$$
g\left(y_{1}, y_{2}\right)=\frac{1}{2 \pi} \cdot \frac{1}{\left(1+y_{1}^{2}+y_{2}^{2}\right)^{3 / 2}} \quad\left(\begin{array}{l}
-\infty<y_{1}<+\infty \\
-\infty<y_{2}<+\infty
\end{array}\right)
$$

Proof. The characteristic function of $\ln \left|X_{k}\right|$ where $X_{k}$ is normal distributed with zero mean and standard deviation $\sigma$ is

$$
\varphi_{k}(t)=E \exp i t \ln \left|X_{k}\right|=(\sigma \sqrt{2})^{i t} \frac{\Gamma((1+i t) / 2)}{\sqrt{\pi}}
$$

The characteristic function of the pair $\left(\ln \left|Y_{2}\right|, \ln \left|Y_{2}\right|\right)$ is

$$
\begin{aligned}
\varphi\left(t_{1}, t_{2}\right) & =\varphi_{1}\left(t_{1}\right) \varphi_{2}\left(t_{2}\right) \varphi_{3}\left(-t_{1}-t_{2}\right) \\
& =\frac{1}{\pi^{3 / 2}} \Gamma\left(\frac{1+i t_{1}}{2}\right) \Gamma\left(\frac{1+i t_{2}}{2}\right) \Gamma\left(\frac{1-i\left(t_{1}+t_{2}\right)}{2}\right) .
\end{aligned}
$$

It is easy to see that the characteristic function of $\left(\ln \left|Y_{1}\right|, \ln \left|Y_{2}\right|\right)$ where $\left(Y_{1} Y_{2}\right)$ is distributed according to the density (26) is also given by the right side of the formula (28). This ends the proof.

THeOREM 4. Let $X_{1}, X_{2}, X_{3}$ be three independent real symmetrical about the origin random variables satisfying the condition $P\left(X_{k}\right.$ $=0)=0(k=1,2,3)$. Denote

$$
V_{1}=\frac{X_{1}}{\sqrt{\overline{X_{1}^{2}+X_{2}^{2}}}}, \quad V_{2}=\frac{\sqrt{X_{1}^{2}+X_{2}^{2}}}{\sqrt{\overline{X_{1}^{2}+X_{2}^{2}+X_{3}^{2}}}}
$$

The necessary and sufficient condition for $X_{k}$ to be normal distri- 
buted with a common standard deviation is that $V_{1}$ and $V_{a}$ are independent distributed according to the densities

$$
\begin{aligned}
& h_{1}(v)= \begin{cases}\frac{1}{\pi} \cdot \frac{1}{\sqrt{1-v^{2}}} & \text { for }|v|<1 \\
0 & \text { elsewhere }\end{cases} \\
& h_{2}(v)= \begin{cases}\frac{v}{\sqrt{1-v^{2}}} & \text { for } 0<v<1 \\
0 & \text { elsewhere. }\end{cases}
\end{aligned}
$$

Proof. The necessary condition is obvious. In order to prove the sufficient condition let us put

$$
V_{1}=\frac{Y_{1}}{\sqrt{Y_{1}^{2}+Y_{2}^{2}}}, \quad V_{2}=\frac{\sqrt{Y_{1}^{2}+Y_{2}^{2}}}{\sqrt{1+Y_{1}^{2}+Y_{2}^{2}}}
$$

where $Y_{1}, Y_{2}$ are given by formula (5). It is easy to see that if $\left(V_{1}, V_{2}\right)$ are independently distributed according to (30), then $\left(Y_{1}, Y_{2}\right)$ is distributed according to the density (26). Using Theorem 3 it is easy to see that all $X_{k}$ are normal distributed with a common standard deviation. This ends the proof.

\section{REFERENCES}

1. I. Kotlarski, On pairs of independent random variables whose quotients follow some known distribution, Colloquium Mathematicum, Vol. IX, 1962, Fasc. 1, 151-162.

2. - On random variables whose quotient follows the Cauchy law, Colloquium Mathematicum, Vol. VII, 1960, Fasc. 2, 278-284.

3. R. G. Laha, An example of a non-normal distribution where the quotient follows the Cauchy law, Proceedings of the National Academy of Sciences 44 (1958), 222-223. 4. - On a class of distribution functions where the quotient follows the Cauchy law, Transactions of the American Mathematical Society 93 (1959), 205-215.

5. - On the laws of Cauchy and Gauss, Ann. Math. Statist. 30 (1959) 1165-1174.

6. - On a problem connected with beta and gamma distributions, Transactions of the American Mathematical Society, Vol. 113, No. 2, November 1964, 287-298.

7. E. Lukacs and R. G. Laha, Application of Characteristic Functions, London, 1964.

8. A. J. Markushevitsh, Teoria analititsheskich funkcji, Moskwa, 1950, (The Theory of Analytical Functions, Moskow, 1950, Russian).

9. J. G. Mauldon, Characterizing properties of statistical distributions, Quarterly Journal of Mathematics, Oxford Series, 2.7, 1956, 155-160.

10. G. P. Steck, A uniqueness property not enjoyed by the normal distribution, Ann. Math. Statist. 29 (1958) 604-606.

Received February 26, 1965.

Politechnika Warszawska, Poland 


\section{PACIFIC JOURNAL OF MATHEMATICS}

H. SAMELSON

Stanford University

Stanford, California

J. P. JaNs

University of Washington

Seattle, Washington 98105

\section{EDITORS}

J. DugundJI

University of Southern California Los Angeles, California 90007

RICHARD ARENS

University of California

Los Angeles, California 90024

\section{ASSOCIATE EDITORS}

E. F. BECKENBACH

B. H. NEUMANN

F. WOLF

K. YosidA

\section{SUPPORTING INSTITUTIONS}

UNIVERSITY OF BRITISH COLUMBIA

CALIFORNIA INSTITUTE OF TECHNOLOGY

UNIVERSITY OF CALIFORNIA

MONTANA STATE UNIVERSITY

UNIVERSITY OF NEVADA

NEW MEXICO STATE UNIVERSITY

OREGON STATE UNIVERSITY

UNIVERSITY OF OREGON

OSAKA UNIVERSITY

UNIVERSITY OF SOUTHERN CALIFORNIA
STANFORD UNIVERSITY

UNIVERSITY OF TOKYO

UNIVERSITY OF UTAH

WASHINGTON STATE UNIVERSITY

UNIVERSITY OF WASHINGTON

AMERICAN MATHEMATICAL SOCIETY CHEVRON RESEARCH CORPORATION TRW SYSTEMS

NAVAL ORDNANCE TEST STATION 


\section{Pacific Journal of Mathematics}

\section{Vol. 20, No. $1 \quad$ September, 1967}

Leonard Daniel Baumert, Extreme copositive quadratic forms. II ........ 1

Edward Lee Bethel, A note on continuous collections of disjoint

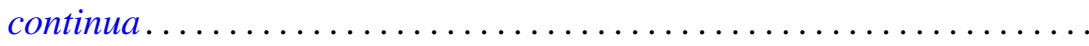

Delmar L. Boyer and Adolf G. Mader, A representation theorem for abelian groups with no elements of infinite p-height ...................

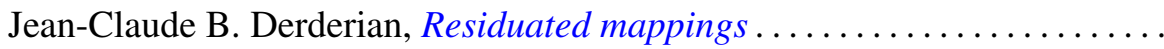

Burton I. Fein, Representations of direct products of finite groups ......... 45

John Brady Garnett, A topological characterization of Gleason parts.......

Herbert Meyer Kamowitz, On operators whose spectrum lies on a circle or

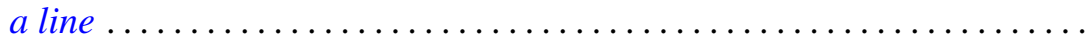

Ignacy I. Kotlarski, On characterizing the gamma and the normal distribution ........................................

Yu-Lee Lee, Topologies with the same class of homeomorphisms ..........

Moshe Mangad, Asymptotic expansions of Fourier transforms and discrete polyharmonic Green's functions ...........................

Jürg Thomas Marti, On integro-differential equations in Banach spaces ....

Walter Philipp, Some metrical theorems in number theory.............. 109

Maxwell Alexander Rosenlicht, Another proof of a theorem on rational

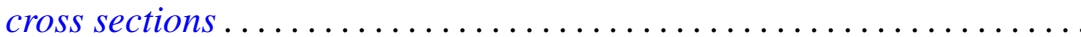

Kenneth Allen Ross and Karl Robert Stromberg, Jessen's theorem on Riemann sums for locally compact groups

Stephen Simons, A theorem on lattice ordered groups, results of Ptak, Namioka and Banach, and a front-ended proof of Lebesgue's theorem...

Morton Lincoln Slater, On the equation $\varphi(x)=\int_{x} x+1 K(\xi) f[\varphi(\xi)] d \xi \ldots$ 155 Arthur William John Stoddart, Existence of optimal controls .. 167 Burnett Roland Toskey, A system of canonical forms for rings on a direct sum of two infinite cyclic groups ....................

Jerry Eugene Vaughan, A modification of Morita's characterization of dimension 